\title{
Enhancement Mode operation in AllnN/GaN (MIS)HEMTs on Si Substrates using Fluorine Implant
}

\author{
Z.H. Zaidi ${ }^{1}$, K.B. Lee ${ }^{1}$, I. Guiney², H. Qian¹, S. Jiang ${ }^{1}$, D.J. Wallis², C.J. \\ Humphreys $^{2}$ and P.A. Houston ${ }^{1}$ \\ ${ }^{1}$ Department of Electronic and Electrical Engineering, The University of Sheffield, Mappin Street Sheffield, S1 3JD, \\ United Kingdom \\ ${ }^{2}$ Department of Materials Science and Metallurgy, The University of Cambridge, 27 Charles Babbage Road \\ Cambridge, CB3 OFS, United Kingdom
}

*corresponding author: zaffar.zaidi@ sheffield.ac.uk

\begin{abstract}
We have demonstrated enhancement mode operation of AlInN/GaN (MIS)HEMTs on Si substrates using the fluorine plasma implant technique. The plasma RF power and treatment time was optimized to prevent the penetration of the fluorine into the channel region to maintain high channel conductivity and transconductance. An analysis of the threshold voltage was carried out which defined the requirement for the fluorine sheet concentration to exceed the charge at the dielectric/AlInN interface to achieve an increase in the positive threshold voltage after deposition of the dielectric. This illustrates the importance of control of both the plasma conditions and the interfacial charge for a reproducible threshold voltage. A positive threshold voltage of $+3 \mathrm{~V}$ was achieved with a maximum drain current of $367 \mathrm{~mA} / \mathrm{mm}$ at a forward gate bias of $10 \mathrm{~V}$.
\end{abstract}

\section{Introduction}

Lattice matched AlInN/GaN HEMTs are competing with conventional AlGaN/GaN HEMTs for RF, microwave frequency and high power amplifier applications [1-2]. One of the advantages of AlInN based HEMTs is the growth of stress free lattice matched structures which can improve device reliability by reducing mechanical strain in the structure. Additionally, high spontaneous polarization charge present in AlInN HEMTs favors high 2DEG density and the thin AlInN barrier offers high transconductance.

Recently there has been intensive work on GaN HEMTs aimed at the development of enhancement mode operation for power control applications. Also, there is interest in developing normally-off GaN based HEMTs for RF, logic and gate drive circuit applications because of simpler circuit configurations, 
reduced circuit size and fail safe operation [3]. Several techniques have been used to achieve enhancement mode operation in $\mathrm{AlGaN} / \mathrm{GaN}$ devices including gate recessing [4], use of p-type cap layers [5], oxidation of the barrier [6], thin barrier structures [7] and incorporation of fluorine ions in the barrier region [8]. Each technology has its own merits and drawbacks and there is no clear winner for the development of the ultimate enhancement mode transistor. AlInN/GaN enhancement mode HEMTs have also been realized using thin barriers [9] and using a GaN/InAlN/GaN structure where the GaN cap reduces the 2DEG density [10]. However, devices with extremely thin barrier layer combinations suffer from high access resistance and may be difficult to reproduce uniformly across a wafer and from wafer to wafer. Recently published work using fluorine based plasma treatment [11] demonstrated a positive shift in the threshold voltage but only a small positive threshold voltage was achieved and attempts to increase the $\mathrm{F}$ dose resulted in reduced 2DEG mobility. About the same time, enhancement mode AlInN/GaN MISHEMTs on sapphire substrates were demonstrated with a threshold voltage of $+1.8 \mathrm{~V}$ [12] but detailed analysis and optimization were lacking in this work. In this paper, we achieved $+3 \mathrm{~V}$ threshold voltage. Our previous recent work on this topic [13] was concentrated on achieving high blocking voltage. In this paper we present an analysis of control of the threshold voltage, studies of subthreshold slope and demonstrate that optimization of plasma exposure in the AlInN/GaN is required to achieve high positive threshold voltage combined with high transconductance (channel mobility). Comparisons are made with AlGaN/GaN HEMTs subject to equivalent fluorine plasma treatment. We achieved normally-off operation in AlInN/GaN HEMTs on 6-inch Si substrates with high positive threshold voltage combined with high transconductance and drain current.

\section{Device Fabrication and Measurement}

Both AlGaN and AlInN HEMT devices were fabricated from wafers grown by Metal Organic Chemical Vapor Deposition on 6-inch Si substrates. To facilitate the growth with lattice mismatched Si substrate a combination of an AlN nucleation layer and graded AlGaN layers were used in both structures. The barrier layer thickness extracted from the $\mathrm{CV}$ measurement for the $\mathrm{Al}_{0.26} \mathrm{Ga}_{0.74} \mathrm{~N}$ HEMT layer was $30 \mathrm{~nm}$ and $13 \mathrm{~nm}$ for the $\mathrm{Al}_{0.83} \mathrm{In}_{0.17} \mathrm{~N}$ HEMT. This includes a $1 \mathrm{~nm}$ mobility enhancement AlN layer present in both structures between the barrier layers and the GaN channel region. Both wafers were also capped with a $2 \mathrm{~nm}$ undoped GaN layer. A standard device fabrication procedure was followed up to the $\mathrm{Ti} / \mathrm{Al} / \mathrm{Ti} / \mathrm{Au}(20 \mathrm{~nm} / 100 \mathrm{~nm} / 45 \mathrm{~nm} / 55 \mathrm{~nm})$ ohmic contact formation step. After this a $1 \mu \mathrm{m}$ gate window was opened by optical lithography and the gate metal placed symmetrically between the source and 
drain contacts (7 $\mu \mathrm{m}$ separation) and then fluorine plasma treatment was carried out in an inductively coupled plasma (ICP) chamber using $\mathrm{CHF}_{3}$ gas. Both high $(150 \mathrm{~W})$ and low $(75 \mathrm{~W}) \mathrm{RF}$ power exposures with the same gas flow and chamber pressure were carried out. The Ni/Au (20 nm/140 nm) self-aligned gate contact was deposited straight after the fluorine treatment using the same gate opening. For the fabrication of the metal insulator semiconductor (MIS) structure, a 25nm Plasma Enhanced Chemical Vapor Deposition (PECVD) $\mathrm{SiN}_{\mathrm{x}}$ layer was deposited before the gate metal. After gate formation, the samples were annealed under nitrogen gas in a rapid thermal annealing chamber at $500^{\circ} \mathrm{C}$ for 5 minutes to recover any plasma induced defects. Finally, Ti/Au $(20 \mathrm{~nm} / 200 \mathrm{~nm})$ pads were deposited to allow electrical probing of the devices. The contact resistance and sheet resistance extracted from transmission line measurements for the AlGaN structure is $0.8 \mathrm{ohm} . \mathrm{mm}$ and $450 \mathrm{ohm} / \mathrm{sq}$, whereas the AlInN structure yielded $0.4 \mathrm{ohm} . \mathrm{mm}$ and $240 \mathrm{ohm} / \mathrm{sq}$ respectively. Room temperature Hall measurements revealed a mobility of $1773 \mathrm{~cm}^{2} \mathrm{~V}^{-1} \mathrm{~s}^{-1}$ and $2 \mathrm{DEG}$ density of $6.8 \times 10^{12} \mathrm{~cm}^{-2}$ in the AlGaN structure and $1717 \mathrm{~cm}^{2} \mathrm{~V}^{-1} \mathrm{~s}^{-1}$ and $1.3 \times 10^{13} \mathrm{~cm}^{-2}$ in the AlInN structure.

\section{Results and Discussions}

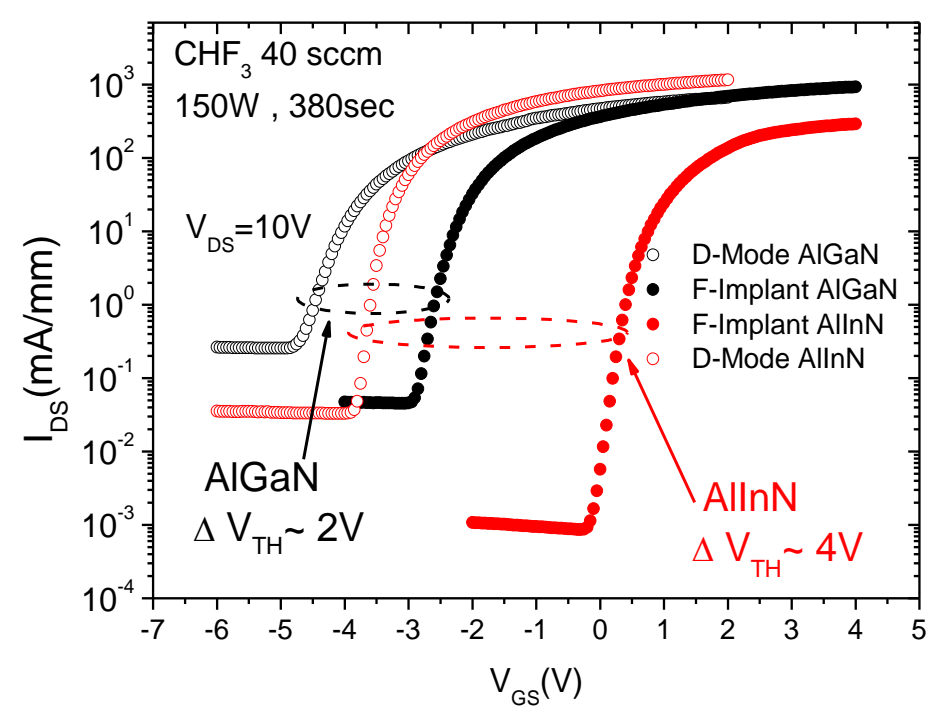

Figure: 1 Gate transfer characteristics of both AlGaN/GaN (black circles) and AlInN/GaN (red circles) HEMTs without (empty circles) and with (filled circles) fluorine implant under the same exposure conditions.

Fluorine ions have been demonstrated to behave as acceptor-like negatively charged particles in the barrier layer which results in 2DEG charge depletion directly beneath the implanted region [8] and a 
positive shift in the threshold voltage. Figure 1 shows the gate transfer characteristics of both $\mathrm{AlGaN} / \mathrm{GaN}$ and AlInN/GaN HEMTs without and with the fluorine implant under the same exposure conditions (plasma exposure time and RF power). It can be seen that, despite having a higher 2DEG concentration, a much larger positive threshold voltage shift $(\sim+4 \mathrm{~V})$ is observed in the AlInN/GaN structure compared to the more conventional AlGaN/GaN HEMTs $(\sim+2 \mathrm{~V})$ for nominally the same dose of fluorine. This observation will be discussed later.

One of the benefits of fluorine implantation in AlGaN HEMTs is the reduction in charge trap density near the barrier surface arising from the plasma processing [14]. This is also observed here in AlInN HEMTs. In the AlInN/GaN HEMT, the sub-threshold slope (S.S.) is reduced from 175 to 121 $\mathrm{mV} / \mathrm{decade}$ with the fluorine treatment (figure 1) which we interpret as resulting from a reduction in the interface trap charge density, $D_{i t}$. Using the following expression [15]

$$
D_{i t}=\left(\frac{S . S .}{\ln (10)} \times \frac{q}{k T}-1\right) \frac{C}{q^{2}}
$$

where $\mathrm{q}$ is the electron charge, $T$ is the absolute temperature in Kelvin, $\mathrm{k}$ is the Boltzmann constant and $C$ is the gate capacitance per unit area, the measured $D_{i t}$ reduced from 8.8 to $4.7 \times 10^{12} \mathrm{~cm}^{2} \mathrm{eV}^{-1}$ following the fluorine treatment. Comparable observations for the AlGaN/GaN HEMT result in a reduction from 13.8 to $6.44 \times 10^{12} \mathrm{~cm}^{2} \mathrm{eV}^{-1}$.

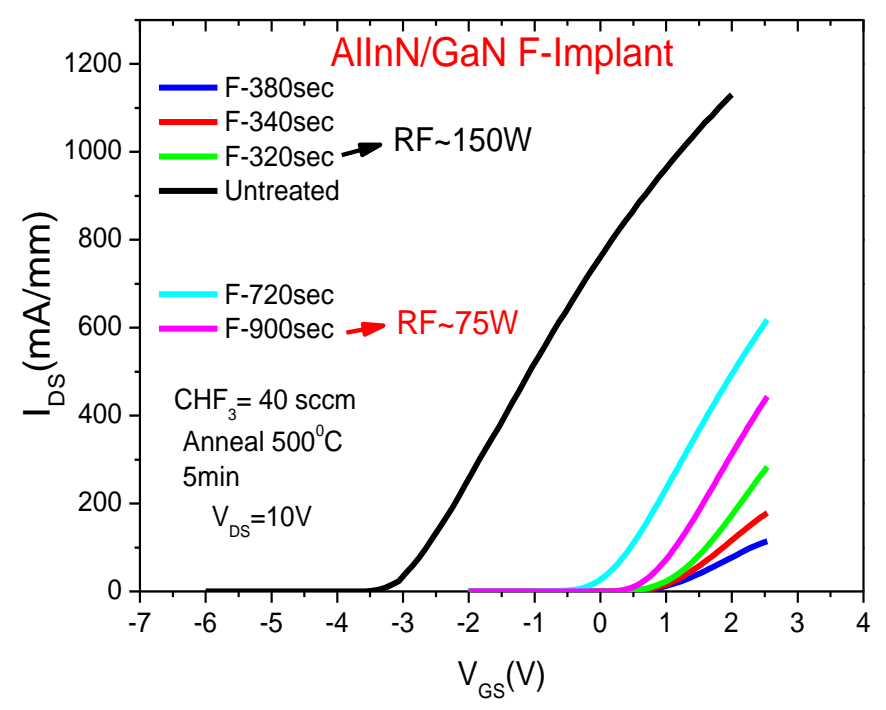

(a)

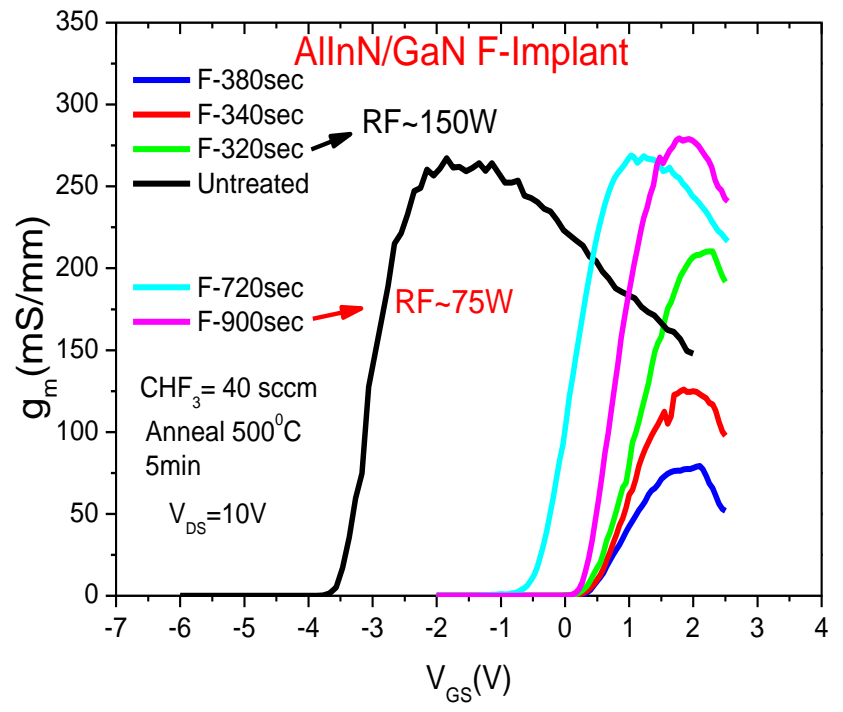

(b)

Figure: 2 (a) Gate transfer characteristics of fluorine implanted AlInN/GaN HEMTs with different exposure times and powers. (b) Transconductance plot of AlInN/GaN HEMTs with varying fluorine exposure time and powers. 
The gate transfer characteristics and transconductance of AlInN/GaN HEMTs after exposure to fluorine plasma at RF powers of $150 \mathrm{~W}$ and $75 \mathrm{~W}$ with different exposure times are shown in figures 2 (a) and (b) respectively. A systematic shift in the gate threshold voltage is observed with an increase in exposure time to fluorine plasma, consistent with an increased fluorine dosage. After the plasma exposure, samples are annealed at $500^{\circ} \mathrm{C}$ for 5 minutes to recover from any plasma induced defects. As shown in figure 2(b) the transconductance does not fully recover after annealing for the $150 \mathrm{~W}$ exposures in the AlInN/GaN devices. This is in contrast to the AlGaN/GaN HEMTs, where full recovery in the transconductance was observed after $500^{\circ} \mathrm{C}$ annealing. However, when the RF power was adjusted to 75 $\mathrm{W}$ with longer exposure times compared to the $150 \mathrm{~W}$ (to offset the reduced flux from lower plasma energy), the transconductance of the AlInN/GaN HEMTs is recovered to the pre-implant values after annealing. We interpret the unrecoverable degradation in transconductance at the higher power $(150 \mathrm{~W})$, and hence higher energy, to the larger number of fluorine ions which have penetrated into the channel region (see figure 3 ), adversely affecting the 2 DEG mobility.

Due to the presence of the thin barrier, AlInN HEMTs can suffer from high gate leakage currents. A beneficial result of the fluorine implant is that the conduction band of the AlInN barrier is bent upward as shown schematically in figure 3 due to the negatively charged fluorine space charge. Consequently, the Schottky barrier height $\left(\phi_{b}\right)$ is effectively increased by $\phi_{F}$, resulting in a suppression of the gate leakage by a factor of 30 . Figure 4 shows the excellent enhancement mode $I V$ characteristics with a positive threshold voltage of $+0.5 \mathrm{~V}$ (defined by extrapolating a straight line from a point of peak transconductance on the drain current to zero), on-resistance, $\mathrm{R}_{\mathrm{ON}} \sim 5 \Omega . \mathrm{mm}$ and a high transconductance of $275 \mathrm{mS} / \mathrm{mm}$.

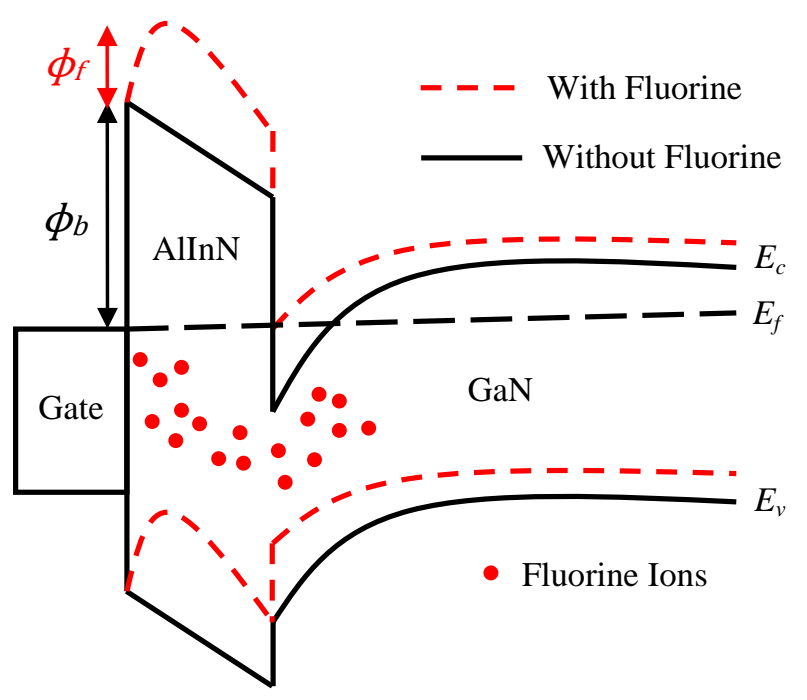

Figure: 3 AlInN/GaN heterostructure 5 band diagram showing penetration of fluorine ions into GaN channel region at RF power of $150 \mathrm{~W}$. 


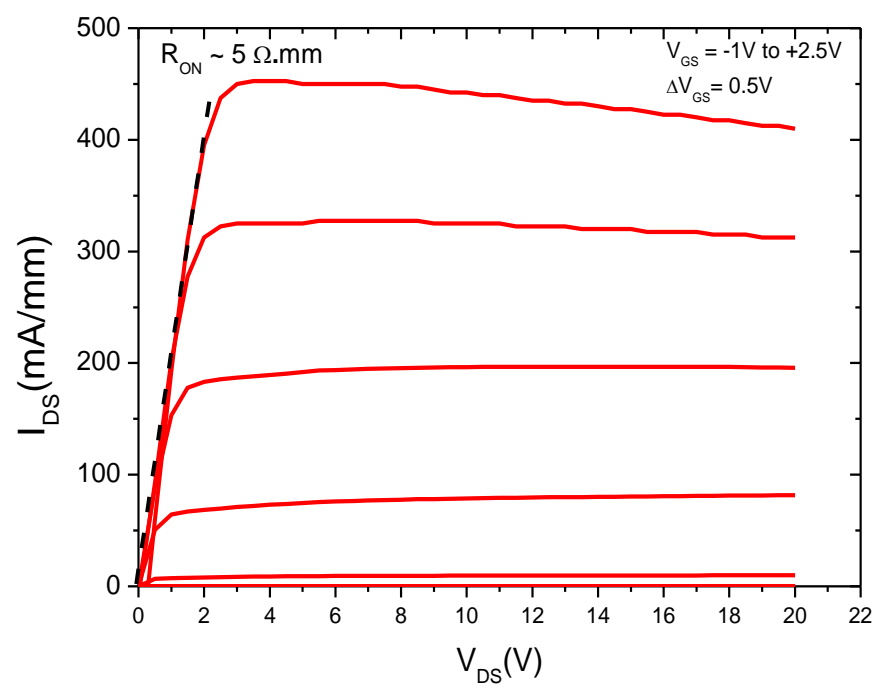

Figure 4: $I V$ characteristics of enhancement mode AlInN/GaN HEMT after fluorine implant at $75 \mathrm{~W}$.

Experimentally we find that, to achieve a positive threshold voltage shift by inserting a dielectric layer between the barrier and gate region, it is imperative to ensure that the HEMT structure receives sufficient fluorine plasma exposure to shift the threshold voltage into the positive region before the dielectric deposition. To understand this, a simplified expression for the threshold voltage was derived by applying Gauss' law at the two charged interfaces of the device to solve for the voltage drops across the dielectric and AlInN barrier shown in figure 3. Setting the 2DEG sheet concentration to $n_{s}=0$ at a gate voltage $V_{g}=V_{t h}$, the threshold voltage is given by

$$
V_{t h(M O S)}=\frac{\emptyset_{B(M O S)}}{q}-\frac{\Delta E_{c(d)}}{q}-\frac{\Delta E_{c(B)}}{q}+\frac{\Delta E_{F}}{q}-\frac{t_{d}}{d} q\left(N_{i n t}-F\right)-\frac{t_{B}}{\epsilon_{B}}(\sigma-q F)
$$

where $\emptyset_{B(\text { MOS })}$ is the metal-dielectric barrier height under the gate, $\mathrm{q}$ is the electron charge, $t_{B}\left(t_{d}\right)$ is the thickness of barrier (dielectric), $\epsilon_{B}\left(\epsilon_{d}\right)$ is the dielectric constant of the barrier (dielectric), $\sigma$ is the total polarisation charge at the barrier/channel interface and $q F$ is the equivalent negative fluorine sheet charge which, when placed at the barrier/buffer interface, properly reflects the voltage change across the barrier and dielectric layers due to the real fluorine distribution in the barrier. $q N_{\text {int }}$ is the nonpolarisation net interface charge between the dielectric and the barrier, $\Delta E_{F}$ is the difference in energy 
between the bottom of the conduction band at the channel and the Fermi level and $\Delta E_{c(B)}\left(\Delta E_{c(d)}\right)$ is the conduction band discontinuity at the hetero-structure (barrier/dielectric) interface.

Examining equation 2, the term containing the dielectric thickness, $t_{d}$, requires the negative fluorine sheet concentration to overcome $q N_{\text {int }}$ for the required positive shift in threshold voltage as the dielectric layer thickness increases. $q N_{i n t}$ represents the total net unbalanced sheet charge, excluding fluorine, and is assumed concentrated at the AlInN barrier/dielectric interface. It can also take account of fixed charge in the dielectric bulk if present. This net charge is taken as positive in order to explain our experimental results. Using equation 2 and the measured voltage shift for the fluorine implant before and after the SiN deposition, values of $1.7 \times 10^{13} \mathrm{~cm}^{-2}$ and $1.28 \times 10^{13} \mathrm{~cm}^{-2}$ were extracted for the equivalent fluorine sheet concentration and dielectric/barrier interfacial positive sheet charge, respectively. Factors affecting this charge include the surface condition of the AlInN barrier and the deposition conditions of the dielectric. In addition, as well as introducing the fluorine charge, the plasma treatment before dielectric deposition may also affect the value of $q N_{\text {int }}$. Therefore, careful control of the barrier surface, the barrier/dielectric interface and the bulk dielectric charge is required in order to achieve reproducible threshold voltages. However, the complete elimination of this interfacial charge would seem to be impossible due to charge balance requirements on formation of the 2DEG.

The large shift in Vth for the AlInN devices compared to the AlGaN devices mentioned earlier is remarkable when considering the larger capacitance (smaller voltage shift expected for the same fluorine charge) together with the higher polarization charge to be overcome in the former case. Details of the fluorine incorporation mechanisms in these systems are lacking, but it may be the case that it incorporates more efficiently in AlInN compared to AlGaN. In addition, maintaining crystal quality in $\mathrm{AlInN}$ is more challenging compared to $\mathrm{AlGaN}$ and this may also be an influencing factor. Future work aims to gain more insight into the fluorine implant mechanisms.

The gate transfer characteristics and transconductance after optimized plasma treatment and deposition of $25 \mathrm{~nm}$ of $\mathrm{SiN}_{\mathrm{x}}$ under the gate show a high positive threshold voltage of $+3 \mathrm{~V}$ (figure 5). The peak transconductance is reduced to $60 \mathrm{mS} / \mathrm{mm}$ compared to that for devices without the dielectric, consistent with the reduced gate capacitance from the increase in overall gate to channel distance. However, the MIS structure combined with the high barrier due to the fluorine space charge, allows a forward gate voltage swing up to $+10 \mathrm{~V}$, producing a maximum drain current of $367 \mathrm{~mA} / \mathrm{mm}$. One of the drawbacks of this structure is the severe hysteresis in drain current observed in transitioning from a high forward gate bias to a reverse bias. This is commonly assigned to electron transfer from the 2DEG into traps at 
the interface between the barrier and dielectric under large forward bias which are subsequently slow to emit once the gate bias is reversed. Hysteresis was not observed in reference [11], most likely due to the limited forward bias achieved $(+5 \mathrm{~V})$.

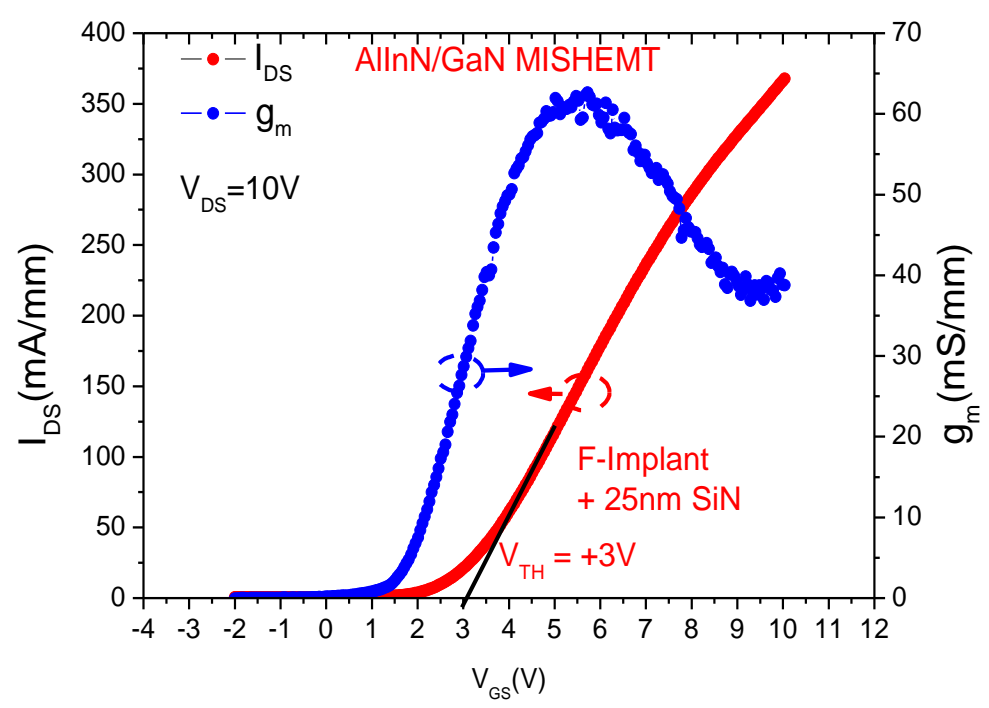

Figure: 5 Gate transfer and transconductance plot of AlInN/GaN MISHEMT with fluorine implant and $25 \mathrm{~nm} \mathrm{SiN}_{\mathrm{x}}$ gate dielectric.

\section{Conclusion}

We have demonstrated enhancement mode operation in AlInN/GaN HEMTs using fluorine ion implantation from plasma treatment. By controlling the fluorine implant to within the narrow AlInN barrier through optimization of the plasma RF power and exposure time, post implantation recovery of the high channel conductivity and transconductance through annealing is possible. The inclusion of $\operatorname{SiN}_{\mathrm{x}}$ under the gate to achieve increased positive threshold voltage requires the fluorine concentration to overcome the net barrier/dielectric interfacial charge, excluding that due to polarization. A reproducible threshold voltage therefore requires close control of both the plasma conditions and the interfacial charge.

\section{Acknowledgements}

The authors acknowledge financial support from the Engineering and Physics Sciences Research Council (EPSRC) under EP/K014471/1 (Silicon Compatible GaN Power Electronics). 


\section{References}

[1] N Sarazin, E Morvan, M di Forte Poisson, M Oualli, C Gaquiere, O Jardel, O Drisse, M Tordjman, M Magis and S L Delage, "AlInN/AIN/GaN HEMT Technology on SiC With 10-W/mm and 50\% PAE at $10 \mathrm{GHz}$,’ IEEE Electron Device Letter, vol. 31, no. 1, pp. 11-13,(2010).

[2] K. Chabak, M. Trejo, A. Crespo, D. Walker, J. Yang, R. Gaska, M. Kossler, J. Gillespie, G. Jessen, V. Trimble, D. Via, "Strained AlInN/GaN HEMTs on SiC with 2.1-A/mm output current and 104Ghz cutoff frequency”, IEEE Electron Device Letters, vol. 31, no 6, pp. 561-563, (2010).

[3] S. Maroldt, C. Haupt, W. Pletschen, S. Müller, R. Quay, O. Ambacher, C. Schippel, and F. Schwierz, "Gate-recessed AlGaN/GaN based enhancement-mode high electron mobility transistors for high frequency operation,” Japanese Journal of Applied Physics, vol. 48, no. 4, pp. 04C083-1-04C0833,(2009).

[4] W. Saito , Y. Takada, M. Kuraguchi , K. Tsuda and I. Omura, "Recessed-gate structure approach toward normally off high-voltage AlGaN/GaN HEMT for power electronics applications," IEEE Transaction on Electron Devices, vol. 53, no. 2, pp. 356 -362, (2006).

[5] Y. Uemoto, M. Hikita, H. Ueno, H. Matsuo, H. Ishida, M. Yanagihara, T. Ueda, T. Tanaka and D. Ueda, "Gate injection transistor (GIT)—A normally-off AlGaN/GaN power transistor using conductivity modulation," IEEE Transaction on Electron Devices, vol. 54, no.12, pp. 3393-3399, (2007).

[6] A Banerjee, S Taking, D Macfarlane, A Dabiran and A Wasige, "Development of enhancement mode AlGaN/GaN MOS-HEMTs using localized gate-foot oxidation, " Proceedings of the $5^{\text {th }}$ European Microwave Integrated Circuits Conference (EuMIC), Paris, pp. 302-305, (2010).

[7] Kai Zhang, Minhan Mi, Yonghe Chen, Mengyi Cao, Chong Wang, Xiaohua Ma, Jincheng Zhang, and Yue Hao, "Normally-Off AlGaN/GaN High Electron Mobility Transistors with Thin and High Al Composition Barrier Layers,” Japanese Journal of Applied Physics, vol. 52, issue 11R, pp. 111001-1111001-4, (2013).

[8] Y. Cai , Y. Zhou , K. M. Lau and K. J. Chen "Control of threshold voltage of AlGaN/GaN HEMTs by fluoride-based plasma treatment: From depletion mode to enhancement mode", IEEE Transactions on Electron Devices, vol. 53, no. 9, pp. 2207 -2215 (2006).

[9] D. Morgan, M. Sultana, H. Fatima, S. Sugiyama, Q. Fareed, V. Adivarahan, M. Lachab and A. Khan, "Enhancement Mode Insulating Gate AlInN/AIN/GaN Heterostructure Field-Effect Transistors with 
Threshold Voltage in Excess of +1.5 V," Applied Physics Express, vol. 4, no. 11, pp. 114101-1114101-3, (2011).

[10] M. Jurkovi`c et al., "Schottky-barrier normally off GaN/InAlN/AlN/GaN HEMT with selectively etched access region," IEEE Electron Device Letters, vol. 34, no. 3, pp. 432-434, (2013).

[11]Zongyang $\mathrm{Hu}$ et al., "Impact of $\mathrm{CF}_{4}$ plasma treatment on threshold voltage and mobility in $\mathrm{Al}_{2} \mathrm{O}_{3} / \mathrm{InAlN} / \mathrm{GaN}$ MOSHEMTs” Applied Physics Express, vol 7, no. 3, pp. 031002-1-031002-4, (2014).

[12] ShengLei Zhao, JunShuai Xue, Peng Zhang, Bin Hou, Jun Luo, XiaoJiao Fan, JinCheng Zhang, XiaoHua $\mathrm{Ma}$ and Yue Hao, "Enhancement-mode $\mathrm{Al}_{2} \mathrm{O}_{3} / \mathrm{InAlN} / \mathrm{AlN} / \mathrm{GaN}$ metal-insulatorsemiconductor high-electron-mobility transistor with enhanced breakdown voltage using fluoridebased plasma treatment," Applied Physics Express, vol. 7, no. 7, pp. 0711002-1-0711002-4, (2014).

[13] Kean B. Lee et al., "Enhancement-mode metal-insulator-semiconductor GaN/AlInN/GaN heterostructure fieldeffect transistors on $\mathrm{Si}$ with a threshold voltage of $+3.0 \mathrm{~V}$ and blocking voltage above 1000 V” Applied Physics Express, vol. 8, no. 3, pp. 036502-1-036502-3, (2015).

[14]C. H. Chen, C. W. Yang, H. C. Chiu, and Jeffrey. S, "Characteristic comparison of AlGaN/GaN enhancement-mode HEMTs with $\mathrm{CHF}_{3}$ and $\mathrm{CF}_{4}$ surface treatment," Journal of Vacuum Science and Technology B, 30, 021201 (2012).

[15] S.M Sze, "Physics of Semiconductor Devices," John Wiley and Sons, 3rd Edition, November, (2006). 